\title{
MEDICINAL FLORA OF THE VIDLIČ MOUNTAIN IN SERBIA
}

\author{
MARIJA MARKOVIĆ ${ }^{\mathbf{1}}$, VESNA STANKOV JOVANOVIĆ ${ }^{\mathbf{1}}$, MIRJANA SMILJIĆ \\ ${ }^{1}$ Faculty of Sciences and Mathematics, University of Niš, Serbia \\ ${ }^{2}$ Faculty of Natural Sciences and Mathematics, University of Priština, Kosovska Mitrovica, Serbia
}

\begin{abstract}
A study of medicinal flora of the Vidlič mountain situated in southeastern Serbia was conducted. The presence of 264 plant species, that are considered official or used in folk medicine is recorded. An overview of medicinal plants is given in a systematic order. For each herb species, main medicinal substances which enter in its chemical composition are listed. An overview of plant parts which are curative and which families contain the highest numbers of medicinal species, are presented. A special review of species that contain toxic substances, as well as species that have become so rare in their natural habitats by irrational exploitation in nature that they have been declared protected and strictly protected taxa in the Republic of Serbia is given.
\end{abstract}

Keywords: Mt. Vidlič, Medicinal plants, Active ingredients, Protected species.

\section{INTRODUCTION}

Vidlič mountain, which is situated in the Pirot's county, according with its position, exerts characteristics of the central Balkan area (Ćirić, 1971).

Within our homeland, Vidlić is peripheral mountain, crossed by Serbian-Bulgarian border. Its long and characteristic ridge, beginning above Pirot city, situated in NW-SE direction till the border with Bulgaria, but there doesn't end, continuing to the Bulgarian territory, all along Sofia (Martinović, 1979-1980).

The mountain Vidlič is a continuous belt of about $13 \mathrm{~km}$ long and of different widths from 250 to $1500 \mathrm{~m}$ (Marinkov, 1999). Its total length is $40 \mathrm{~km}$ according to Anđelković \& Nikolić (1958).

The westernmost point of the study area on the river Temštica has coordinates: $43^{\circ} 122^{\prime}$ ' ' north geographical latitude and $22^{\circ} 33^{\prime} 4^{\prime}$ ' east geographical longitude, and the easternmost point close to the border with Bulgaria at village Vlkovija has coordinates: $43^{\circ} 5^{\prime} 5^{\prime}$ ' north geographical latitude and $22^{\circ} 55^{\prime} 1$ ', east geographical longitude (according to Greenwich).

Vidlič Mountain is a branch of Stara planina Mountains according to one group of authors (Mišić et al., 1978). Second group of authors (Vidanović, 1955; Martinović, 1979-1980) things that Vidlič has a separate orographic and tectonic whole, which is completely different from Stara Planina Mountains and Visok.

In geological term the mountain is almost completely built of limestone formations, with the best represented cretaceous and triassic layers.

The prevailing soil types are rendzina and calcomelanosol, which achieve different evolutionary forming phases (Antonović \& Mrvić, 2008).

\footnotetext{
*Coresponding author: marijam@pmf.ni.ac.rs
}

The climate is temperate continental, with hot summers and prominent drought periods, while the upper part of the mountain has features of a mountain climate (Ćirić, 1989).

Deciduous forest of hungarian and turkish oak (Quercetum frainetto-cerris) at low altitudes $500-600 \mathrm{~m}$, as one of the most widespreaded, considered to be climax in the region (Mišić et al., 1978). Different degradation stages of this community and its derivates with oriental hornbeam (Carpinus orientalis) are well represented, in a larger extent on the mountain. Transitional belt between the oak (Quercetum montanum) and beech forests was noticed at upper altitudes $700-900 \mathrm{~m}$. The upper region of the mountain is covered by moesian beech forests (Fagetum montanum moesiacum), considered to be climate-regional community, over $1000 \mathrm{~m}$ above sea level, to the highest altitude of the mountain, which is $1413 \mathrm{~m}$ (Marković et al., 2018). After the clear-cutting, and period of subsequent grazing, many types of meadows, xeric pastures and schrub-like vegetation of the rocky slopes were formed over the vast area of eroded slopes.

\section{THEORETICAL PART}

The medicinal flora of the Vidlič Mountain has not been recorded and systematized yet.

The area of Pirot county was visited by our famous botanist Josif Pančić at the end of the XIX century. The first data of Vidlič flora originated since then and refer on the species which Pančić mentioned for Vidlič in the papers: "Flora Kneževine Srbije" and "Dodatak Flori Kneževine Srbije" (Marković, 2013). More intense study of plants of this region was performed by Lujo Adamović (Marković, 2013), in whose papers Vidlič is often mentioned.

Ranđelović et al. (1991) were studied the medicinal plants of Pirot caunty, and they were recorded the part of the medicinal plant from the Vidlič Mountain. In the monography „Lekovite biljke SR Srbije“ Sarić, ed. (1989) mentioned which plant species were found on Vidlič Mt. 
Aromatic flora of the Vidlilč Mountain was especially systematized, where it was noticed 60 species with essential oil content (Marković, 2006; Marković et al,, 2009). During the research of the resources of medicinal plants in the Pirot county, Marković et al. (2010a, 2010b) were noticed data of medicinal plants which are recorded on the Vidlič Mountain.

\section{EXPERIMENTAL}

\section{Materials and methods}

Field studies of medicinal flora of the Vidlič Mountain were carried out in the period 2002-2014. The list of medicinal flora was made according to the systematic order of the species according to Josifović (1970-1977). Only native representatives of the flora were included in the list of medicinal plants (Table1).
Some additional allochthonous (introduced, invasive and grown in the gardens) representatives of the flora were not included.

A detailed investigation of the medicinal flora included the area of mountain Vidlič in southeastern Serbia, south of the river Visočica and north of the river Nišava, east of the river Temštica and west of the border with Bulgaria. The result of field research was the plant material that was collected, herbarized, labeled and deposited in the "Herbarium Moesiacum Niš", Faculty of Science and Mathematics, University of Niš (HMN).

Identification of the collected plant material was performed according to the "Flora of SR Serbia" (Josifović, 1970-1977). The nomenclature is adjusted according to the "Flora Europaea" (Tutin, 1964-1980; 1993). The belonging to the group of medicinal plants was determined according to Sarić, ed. (1989).

Table 2. The list of medicinal plants of the Vidlič Mountain.

\begin{tabular}{|c|c|c|c|}
\hline Family & Taxon & Compounds & Herbal drug \\
\hline Equisetaceae & Equisetum arvense L. & mineral substances, flavonoids & Herba \\
\hline Aspidiaceae & Dryopteris filix-mas (L.) Schott ! & $\begin{array}{c}\text { floroglucyne derivatives, tannins, fatty } \\
\text { oil }\end{array}$ & Rhizome \\
\hline Polypodiaceae & Polypodium vulgare L. & $\begin{array}{c}\text { tannins, resin, fatty oil, saponins, } \\
\text { sugars }\end{array}$ & Rhizome \\
\hline \multirow[t]{2}{*}{ Pinaceae } & Abies alba Miller & essential oil & $\begin{array}{l}\text { acicula, strobuli, } \\
\text { cortex }\end{array}$ \\
\hline & Pinus nigra Arnold & turpentine & Turio \\
\hline Cupressaceae & Juniperus communis L. * & essential oil, tannins, flavonoids & fructus, lignum \\
\hline Aristolochiaceae & Asarum europaeum L. *! & $\begin{array}{l}\text { essential oil, tannins, glycosides, } \\
\text { alkaloids }\end{array}$ & $\begin{array}{l}\text { herba cum } \\
\text { radic ibus }\end{array}$ \\
\hline \multirow[t]{13}{*}{ Ranunculaceae } & Helleborus odorus Waldst. \& Kit. ! & cardiotonic heterosides, saponins & rhizoma et radix \\
\hline & Nigella arvensis L. & essential oil, saponins, pfatty oil & Semen \\
\hline & Nigella damascena L. & essential oil, saponins, fatty oil & Semen \\
\hline & Aquilegia vulgaris L. & cyanogenetic heterosides, vitamin $\mathrm{C}$ & $\begin{array}{l}\text { semen, folium, } \\
\text { flos }\end{array}$ \\
\hline & Hepatica nobilis Schreber*? & tannins, saponins & herba, folium \\
\hline & $\begin{array}{l}\text { Pulsatilla montana (Hoppe) Reichenb. } \\
\text { subsp. bulgarica Rummelsp. **! }\end{array}$ & saponins, tannins, resin & herba recens \\
\hline & Clematis vitalba $\mathrm{L}$ & glycosides, saponins & Herba \\
\hline & Clematis recta $\mathrm{L}$. & glycosides, saponins & Herba \\
\hline & Ranunculus ficaria L. & mucus, essential oil, saponins & Tuber \\
\hline & Ranunculus repens L. ! & anemone-camphor, saponins & Herba \\
\hline & Ranunculus acris L. ! & anemone-camphor, saponins & Herba \\
\hline & Ranunculus bulbosus L.! & anemone-camphor, saponins & Herba \\
\hline & Adonis vernalis L. **! & cardiotonic glycosides & Herba \\
\hline Berberidaceae & Berberis vulgaris L. & alkaloids, tannins, vitamin $\mathrm{C}$ & $\begin{array}{l}\text { cortex, radix, } \\
\text { folium, fructus }\end{array}$ \\
\hline \multirow[t]{6}{*}{ Papaveraceae } & Papaver dubium L. ! & alkaloids & Flos \\
\hline & Papaver rhoeas L.! & alkaloids, anthocyanins, mucus & Flos \\
\hline & Chelidonium majus L. & alkaloids, flavonoids, saponins & radix, herba \\
\hline & $\begin{array}{l}\text { Corydalis cava (L.) Schweigger \& Körte } \\
\text { subsp. marschalliana (Willd.) Hayek ! }\end{array}$ & alkaloids & Tuber \\
\hline & Corydalis solida (L.) Clairv. ! & alkaloids & Tuber \\
\hline & Fumaria officinalis L. ! & alkaloids & Herba \\
\hline Ulmaceae & Ulmus glabra Hudson & tannins, mucus, bitter substance & Cortex \\
\hline
\end{tabular}




\begin{tabular}{|c|c|c|c|}
\hline Family & Taxon & Compounds & Herbal drug \\
\hline Moraceae & Morus alba $\mathrm{L}$. & vitamins, pectins, rubbers & $\begin{array}{l}\text { folium, fructus } \\
\text { recens }\end{array}$ \\
\hline Cannabaceae & Humulus lupulus L. & resin, tannins, essential oil, flavonoids & Strobuli \\
\hline Urticaceae & Urtica dioica $\mathrm{L}$. & vitamins, tannins & $\begin{array}{l}\text { folium, radix, } \\
\text { semen }\end{array}$ \\
\hline \multirow[t]{5}{*}{ Fagaceae } & Fagus moesiaca (K.Maly) Czech. & $\begin{array}{l}\text { phenolic derivatives, hydrocarbons, } \\
\text { organic acids, resin }\end{array}$ & cortex \\
\hline & Quercus cerris L. & tannins, catechin, flavonoids & cortex \\
\hline & Quercus petraea (Mattuschka) Liebl. & tannins, catechin, flavonoids & cortex \\
\hline & Quercus frainetto Ten. & tannins, catechin, flavonoids & cortex \\
\hline & Quercus pubescens Willd. & tannins, catechin, flavonoids & cortex \\
\hline Betulaceae & Betula pendula Roth. * & $\begin{array}{l}\text { flavonoids, tannins, resin, saponins, } \\
\text { essential oil }\end{array}$ & gemmae, folium \\
\hline \multirow[t]{2}{*}{ Corylaceae } & Corylus colurna L.* & tannins, flavonoids, fatty oil & $\begin{array}{l}\text { cortex, folium, } \\
\text { semen }\end{array}$ \\
\hline & Corylus avelana $\mathrm{L}$. & tannins, flavonoids, fatty oil & $\begin{array}{l}\text { cortex, folium, } \\
\text { semen }\end{array}$ \\
\hline Juglandaceae & Juglans regia $\mathrm{L}$. & $\begin{array}{l}\text { tannins, nafttchynone derivatives, } \\
\text { flavonoids, vitamin } \mathrm{C} \text {, essential oil }\end{array}$ & folium, fructus \\
\hline \multirow[t]{3}{*}{ Caryophyllaceae } & Herniaria glabra L. * & $\begin{array}{c}\text { saponins, flavonoids, coumarins, } \\
\text { tannins }\end{array}$ & herba \\
\hline & Herniaria hirsuta L. * & $\begin{array}{l}\text { saponins, flavonoids, coumarins, } \\
\text { tannins }\end{array}$ & herba \\
\hline & Saponaria officinalis $\mathrm{L}$. & saponins, sugars & radix, herba \\
\hline \multirow[t]{5}{*}{ Polygonaceae } & Polygonum aviculare $\mathrm{L}$. & flavonoids, tannins, mucus & herba \\
\hline & Persicaria hydropiper (L.) Delarbre & flavonoids, tannins, organic acids & herba \\
\hline & Rumex crispus $\mathrm{L}$. & calcium oxalate & radix, folium \\
\hline & Rumex sanguineus L. & calcium oxalate & radix, folium, \\
\hline & Rumex acetosa $\mathrm{L}$. & calcium oxalate & radix, folium \\
\hline \multirow[t]{2}{*}{ Paeoniaceae } & Paeonia tenuifolia L. **! & alkaloids, tannins & flos, radix \\
\hline & Paeonia peregrina Miller **! & alkaloids, tannins & flos, radix \\
\hline \multirow[t]{3}{*}{ Hypericaceae } & Hypericum hirsutum $\mathrm{L}$. & flavonoids, tannins, essential oil & herba \\
\hline & Hypericum perforatum L. * & flavonoids, tannins, essential oil & herba \\
\hline & Hypericum barbatum Jacq. * & flavonoids, tannins, essential oil & herba \\
\hline \multirow[t]{2}{*}{ Vioaceae } & Viola odorata L. $*$ & saponins, bitter substance, mucus & $\begin{array}{l}\text { folium, flos, } \\
\text { radix }\end{array}$ \\
\hline & Viola tricolor $\mathrm{L}$. & mucus, tannins, flavonoids & herba \\
\hline \multirow[t]{7}{*}{ Brassicaceae } & Sisymbrium officinale (L.) Scop. & sulfuric glycosides, vitamin $\mathrm{C}$ & $\begin{array}{l}\text { herba recens, } \\
\text { folium recens }\end{array}$ \\
\hline & Nasturtium officinale $\mathrm{R} . \mathrm{Br}$. & sulfuric heterosides, vitamins & $\begin{array}{l}\text { herba recens, } \\
\text { folium recens }\end{array}$ \\
\hline & Cardamine pratensis $\mathrm{L}$. & sulfuricglycosides, vitamin $\mathrm{C}$, carotene & folium \\
\hline & Cardamine impatiens $\mathrm{L}$. & $\begin{array}{l}\text { sulfuric glycosides, vitamin } \mathrm{C}, \\
\text { carotene }\end{array}$ & stipes, folium \\
\hline & Cardamine flexuosa With. & sulfuricglycosides, vitamin $\mathrm{C}$, carotene & stipes, folium \\
\hline & Capsella bursa-pastoris (L.) Medicus & flavonoids, tannins & herba \\
\hline & Sinapis arvensis $\mathrm{L}$. & fatty oil, sinapin & semen \\
\hline \multirow[t]{2}{*}{ Resedaceae } & Reseda luteola L. & flavonoids, sulfuric glycosides & herba \\
\hline & Reseda lutea $\mathrm{L}$. & flavonoids, sulfuric glycosides & herba \\
\hline \multirow[t]{5}{*}{ Salicaceae } & Populus tremula L. & flavonoids, salicin & cortex, gemmae \\
\hline & Populus nigra L. & resin, flavonoids & cortex, gemmae \\
\hline & Salix alba $\mathrm{L}$. & glycosides, flavonoids, tannins & cortex \\
\hline & Salix cinerea $\mathrm{L}$. & glycosides, flavonoids, tannins & cortex \\
\hline & Salix caprea $\mathrm{L}$. & glycosides, flavonoids, tannins & cortex \\
\hline
\end{tabular}




\begin{tabular}{|c|c|c|c|}
\hline Family & Taxon & Compounds & Herbal drug \\
\hline & Salix purpurea L. & glycosides, flavonoids, tannins & cortex \\
\hline \multirow[t]{5}{*}{ Primulaceae } & Lysimachia nummularia $\mathrm{L}$. & bitter substance, tannins, saponins & herba \\
\hline & Lysimachia punctata $\mathrm{L}$. & tannins, saponins, flavonoids & herba \\
\hline & Lysimachia vulgaris L. & tannins, saponins, flavonoids & herba \\
\hline & Anagallis arvensis L. ! & saponins, tannins & herba \\
\hline & Primula veris L. * & saponins, glycosides, starch, tannins & $\begin{array}{l}\text { flos, rhizoma } \\
\text { cum radicibus }\end{array}$ \\
\hline Tiliaceae & Tilia tomentosa Moench. * & mucus, tannins, mannitol & flos cum bracteis \\
\hline \multirow[t]{2}{*}{ Malvaceae } & Malva sylvestris $\mathrm{L}$. & mucus, tannins, anthocyanins & folium, flos \\
\hline & Malva neglecta Wallr. & mucus, tannins, anthocyanins & folium, flos \\
\hline \multirow[t]{3}{*}{ Euphorbiaceae } & Euphorbia helioscopia L. ! & bitter - hot substances & semen, succus \\
\hline & Euphorbia cyparissias L.！ & bitter - hot substances & semen, succus \\
\hline & Euphorbia amygdaloides L. ! & bitter - hot substances & semen \\
\hline Thymeleaceae & Daphne mezereum L. ! & $\begin{array}{l}\text { resin, flavonoids, glycosides, } \\
\text { coumarins }\end{array}$ & cortex \\
\hline \multirow[t]{23}{*}{ Rosaceae } & Filipendula vulgaris Moench & essential oil, tannins, & $\begin{array}{l}\text { herba, folium, } \\
\text { rhizoma }\end{array}$ \\
\hline & Filipendula ulmaria (L.) Maxim. & essential oil, tannins, glycosides & folium \\
\hline & Rubus idaeus $\mathrm{L}$. & tannins, organic acids, flavonoids & $\begin{array}{l}\text { folium, fructus } \\
\text { recens }\end{array}$ \\
\hline & Rubus caesius $\mathrm{L}$. & tannins, organic acids, flavonoids & folium \\
\hline & Rosa canina L. * & $\begin{array}{c}\text { vitamins, pectins, tannins, glycosides, } \\
\text { organic acids }\end{array}$ & fructus \\
\hline & Agrimonia eupatoria Ledeb. & tannins, bitter substance & herba \\
\hline & Sanguisorba officinalis L. & tannins, starch, saponins & $\begin{array}{l}\text { rhizoma cum } \\
\text { radicibus }\end{array}$ \\
\hline & Sanguisorba minor Scop. & tannins, starch, saponins, sterols & $\begin{array}{l}\text { rhizoma cum } \\
\text { radicibus, herba }\end{array}$ \\
\hline & Geum urbanum L. & tannins, flavonoids & $\begin{array}{l}\text { rhizoma cum } \\
\text { radicibus }\end{array}$ \\
\hline & Potentilla recta $\mathrm{L}$. & tannins, resin & rhizoma \\
\hline & Potentilla erecta $($ L.) Räuschel * & $\begin{array}{l}\text { tannins, saponins, resin, starch, } \\
\text { flobaphens }\end{array}$ & rhizoma \\
\hline & Potentilla reptans $\mathrm{L}$. & tannins, resin & rhizoma \\
\hline & Fragaria vesca L. * & tannins, flavonoids & folium, fructus \\
\hline & Alchemilla sp. div. * & tannins, bitter substance & herba, folium \\
\hline & Cydonia oblong a Miller & $\begin{array}{c}\text { organic acids, vitamin } \mathrm{C}, \text { tannins, } \\
\text { mucu }\end{array}$ & $\begin{array}{l}\text { folium, fructus, } \\
\text { semen }\end{array}$ \\
\hline & Malus sylvestris Miller & vitamins, iodine & fructus, semen \\
\hline & Sorbus aucuparia L. & tannins, vitamins, organic acids & fructus \\
\hline & Sorbus torminalis (L.) Crantz & tannins, vitamins, organic acids & fructus \\
\hline & Sorbus aria (L.) Crantz & tannins, vitamins, rubbers & fructus \\
\hline & Crataegus monogyna Jacq. * & flavonoids & $\begin{array}{l}\text { flos, folium, } \\
\text { fructus }\end{array}$ \\
\hline & Crataegus laevigata (Poiret) DC. ${ }^{*}$ & flavonoids & $\begin{array}{l}\text { flos, folium, } \\
\text { fructus }\end{array}$ \\
\hline & Prunus spinosa $\mathrm{L}$. & $\begin{array}{l}\text { flavonoids, glycosides; tannins, } \\
\text { anthocyanins, pectins }\end{array}$ & flos, fructus \\
\hline & Prunus avium L. & glycosides & fructus, petiolus \\
\hline Grossulariaceae & Ribes uva-crispa L. * & vitamins, carotenes & folium, fructus \\
\hline \multirow[t]{2}{*}{ Crassulaceae } & Hylotelephium telephium (L.) Ohba & $\begin{array}{l}\text { alkaloids, flavonoids, tannins, organic } \\
\text { acids }\end{array}$ & herba recens \\
\hline & Sedum acre L. & $\begin{array}{l}\text { alkaloids, flavonoids, tannins, organic } \\
\text { acids }\end{array}$ & herba recens \\
\hline Fabaceae & Ononis spinosa $\mathrm{L}$. & glycosides, triperpens, essential oil & radix \\
\hline
\end{tabular}




\begin{tabular}{|c|c|c|c|}
\hline Family & Taxon & Compounds & Herbal drug \\
\hline & Medicago lupulina $\mathrm{L}$. & vitamins & folium \\
\hline & Medicago falcata $\mathrm{L}$. & vitamins & folium \\
\hline & Melilotus officinalis (L.) Pallas & coumarins, flavonoids, tannins & herba \\
\hline & Anthyllis vulneraria L. * & tannins, saponins, flavonoids & flos, herba \\
\hline & Genista tinctoria $\mathrm{L}$. & alkaloids, flavonoids, essential oil & herba \\
\hline & Coronilla varia L. ! & heterosides & herba \\
\hline & Coronilla scorpioides (L.) Koch ! & heterosides & herba \\
\hline Lythraceae & Lythrum salicaria $\mathrm{L}$ & glycosides, tannins, flavonoids, mucus & herba \\
\hline \multirow[t]{2}{*}{ Oenotheraceae } & Epilobium parviflorum Schreber * & tannins, flavonoids, mucus & herba, folium \\
\hline & Epilobium angustifolium $\mathrm{L}$. & tannins, flavonoids, mucus & herba, folium \\
\hline Anacard iaceae & Cotinus coggygria Scop. ! & tannins, flavonoids & folium \\
\hline Rutaceae & Dictamnus albus L. & essential oil, alkaloids, bitter substance & herba, radix \\
\hline \multirow[t]{2}{*}{ Aceraceae } & Acer tataricum $\mathrm{L}$. & tannins & cortex, folium \\
\hline & Acer platanoides $\mathrm{L}$. & glycosides & succus \\
\hline Zygophyllaceae & Tribulus terrestris L. & saponins, flavonoids & herba, fructus \\
\hline Oxalidaceae & Oxalis acetosella $\mathrm{L} . !$ & flavone heterosides, oxalyc acid & folium \\
\hline \multirow[t]{3}{*}{ Geraniaceae } & Geranium macrorrhizum L. * & $\begin{array}{l}\text { essential oil, tannins, flavonoids, } \\
\text { pectins, rubbers }\end{array}$ & rhizoma \\
\hline & Geranium robertianum L. * & $\begin{array}{c}\text { tannins, flavonoids, resin, organic } \\
\text { acids }\end{array}$ & herba \\
\hline & Erodium cicutarium (L.) L'Hér. & tannins, flavonoids & herba \\
\hline \multirow[t]{2}{*}{ Polygalaceae } & Polygala amara $\mathrm{L}$. & saponins, bitter substance & herba \\
\hline & Polygala vulgaris $\mathrm{L}$. & saponins, bitter substance & herba \\
\hline \multirow[t]{2}{*}{ Cornaceae } & Cornus mas L. * & tannins, pectins, organic acids & $\begin{array}{l}\text { cortex, folium, } \\
\text { fructus }\end{array}$ \\
\hline & Cornus sanguinea $\mathrm{L}$. & fatty oil & semen \\
\hline Araliaceae & Hedera helix L. ! & saponins & $\begin{array}{l}\text { folium, stipes, } \\
\text { cortex, fructus }\end{array}$ \\
\hline \multirow[t]{11}{*}{ Apiaceae } & Sanicula europaea L. & bitter substance, tannins, saponins & radix, herba \\
\hline & Eryngium campestre L. & saponins, tannins, essential oil & radix \\
\hline & Carum carvi $\mathrm{L}$. & $\begin{array}{l}\text { essential oil, fatty oil, proteins, } \\
\text { carbohydrates }\end{array}$ & fructus \\
\hline & Pimpinella saxifraga $\mathrm{L}$. & $\begin{array}{l}\text { essential oil, coumarins, saponins, } \\
\text { tannins }\end{array}$ & radix \\
\hline & Aegopodium podagraria $\mathrm{L}$. & essential oil, vitamin C & herba \\
\hline & Angelica sylvestris $\mathrm{L}$. & essential oil, res in, bitter substance & radix, fructus \\
\hline & Peucedanum longifolium Waldst. \& Kit. & bitter substance, essential oil & herba, radix \\
\hline & $\begin{array}{l}\text { Pastinaca sativa L. ssp. urens (Req. ex } \\
\text { Godron) Čelak. }\end{array}$ & $\begin{array}{l}\text { essential oil, fatty oil, coumarins, } \\
\text { flavonoids }\end{array}$ & fructus, radix \\
\hline & Heracleum sphondylium L. & essential oil, arginine & $\begin{array}{l}\text { radix, folium, } \\
\text { flos }\end{array}$ \\
\hline & Daucus carota $\mathrm{L}$. & $\begin{array}{c}\text { carotenes, vitamins, pectins; essential } \\
\text { oil, fatty oil }\end{array}$ & radix, fructus \\
\hline & Conium maculatum L. ! & alkaloids & $\begin{array}{l}\text { folium, herba, } \\
\text { fructus }\end{array}$ \\
\hline Celastraceae & Evonymus europaeus L.! & alkaloids & $\begin{array}{l}\text { cortex, stipes, } \\
\text { folium, fructus }\end{array}$ \\
\hline Rhamnaceae & Rhamnus cathartica $\mathrm{L}$. & $\begin{array}{l}\text { anthraquinones, flavonoids, pectins, } \\
\text { glycosides, mucus }\end{array}$ & fructus \\
\hline \multirow[t]{2}{*}{ Apocynaceae } & Vinca minor $\mathrm{L}$. & alkaloids, flavonoids & folium, herba \\
\hline & Vinca herbacea Waldst. \& Kit. ** & alkaloids, flavonoids & folium, herba \\
\hline Asclepiadaceae & Vincetoxicum hirundinaria Medicus ! & toxic compounds (vincetoxin), resin & rhizoma \\
\hline \multirow[t]{2}{*}{ Gentianaceae } & Centaurium erythraea Rafin. * & glycosides, flavonoids & herba \\
\hline & Gentiana cruciata L. * & bitter substance, tannins, glycosides & herba \\
\hline
\end{tabular}




\begin{tabular}{|c|c|c|c|}
\hline Family & Taxon & Compounds & Herbal drug \\
\hline \multirow[t]{3}{*}{ Oleaceae } & Fraxinus ornus $\mathrm{L}$. & $\begin{array}{c}\text { sugars (mannitol), mineral substances, } \\
\text { resin }\end{array}$ & succus (manna) \\
\hline & Fraxinus excelsior $\mathrm{L}$. & tannins, flavonoids, coumarins, resin & $\begin{array}{l}\text { cortex, folium, } \\
\text { semen }\end{array}$ \\
\hline & Ligustrum vulgare L. ! & tannins, essential oil & $\begin{array}{l}\text { cortex, folium, } \\
\text { fructus }\end{array}$ \\
\hline Menyanthaceae & Menyanthes trifoliata L.** & heterosides, tannins & folium \\
\hline \multirow[t]{2}{*}{ Rubiaceae } & Galium odoratum (L.) Scop. * & $\begin{array}{c}\text { coumarin heterosides, bitter substance, } \\
\text { tannins }\end{array}$ & herba \\
\hline & Galium verum $\mathrm{L}$. & glycosides, tannins, saponins & herba \\
\hline \multirow[t]{3}{*}{ Caprifoliaceae } & Viburnum opulus L. & saponins, resin, tannins, glycosides & cortex, fructus \\
\hline & Sambucus ebulus L. & $\begin{array}{c}\text { cyanogenetic heterosides, bitter } \\
\text { substance }\end{array}$ & $\begin{array}{l}\text { radix, folium, } \\
\text { fructus }\end{array}$ \\
\hline & Sambucus nigra $\mathrm{L}$. & $\begin{array}{l}\text { mucus, flavonoids, essential oil, } \\
\text { tannins }\end{array}$ & flos, fructus \\
\hline Valerianaceae & Valeriana officinalis L. & essential oil, tannins, starch & $\begin{array}{l}\text { rhizoma cum } \\
\text { radicibus }\end{array}$ \\
\hline \multirow[t]{2}{*}{ Convolvulaceae } & Cuscuta europaea $\mathrm{L}$. & not studied & herba \\
\hline & Cuscuta epithimum (L.) L. & not studied & herba \\
\hline \multirow[t]{4}{*}{ Boraginaceae } & Cynoglossum officinale $\mathrm{L}$. & alcaloids, tannins & herba, radix \\
\hline & Symphytum officinale L. * & mucus, tannins, starch & radix \\
\hline & Anchusa officinalis L. & alkaloids, tannins, mucus & herba \\
\hline & Pulmonaria officinalis L. * & $\begin{array}{l}\text { mucus, mineral substances, tannins, } \\
\text { flavonoids }\end{array}$ & herba \\
\hline \multirow[t]{5}{*}{ Solanaceae } & Atropa bella-donna L. ! & alkaloids & radix, folium \\
\hline & Hyoscyamus niger L. ! & alkaloids & folium, semen \\
\hline & Solanum dulcamara L. ! & alkaloids, glycosides, tannins & stipes \\
\hline & Solanum nigrum L. ! & alkaloids, tannins, saponins & herba, fructus \\
\hline & Datura stramonium L. ! & alkaloids & folium, semen \\
\hline \multirow[t]{10}{*}{ Scrophulariaceae } & Verbascum phlomoides L. & flavonoids, saponins, mucus & flos \\
\hline & Verbascum phoeniceum $\mathrm{L}$. & flavonoids, saponins & flos \\
\hline & Linaria vulgaris Miller ! & alkaloids, glycosides & herba \\
\hline & Scrophularia nodosa L.! & glycosides, tannins & herba \\
\hline & Veronica officinalis L. * & $\begin{array}{c}\text { flavonoids, glycosides, tannins, } \\
\text { organic acids }\end{array}$ & herba \\
\hline & Veronica beccabunga $\mathrm{L}$. & flavonoids, glycosides & herba recens \\
\hline & Digitalis ferruginea L.! & $\begin{array}{l}\text { cardiotonic glycosides, saponins, } \\
\text { flavonoids }\end{array}$ & folium \\
\hline & Digitalis lanata Ehrh. ! & $\begin{array}{l}\text { cardiotonic glycosides, saponins, } \\
\text { flavonoids }\end{array}$ & folium \\
\hline & Euphrasia rostkoviana Hayne $*$ & glycosides, tannins, resin & herba \\
\hline & $\begin{array}{c}\text { Euphrasia stricta D. Wolff ex J. F. } \\
\text { Lehm. * }\end{array}$ & glycosides, tannins, resin & herba \\
\hline \multirow[t]{3}{*}{ Plantaginaceae } & Plantago major $\mathrm{L}$. & glycosides, tannins, mucus & folium \\
\hline & Plantago media L. & glycosides, tannins, mucus & folium \\
\hline & Plantago lanceolata $\mathrm{L}$. & glycosides, tannins, mucus, flavonoids & folium \\
\hline Verbenaceae & Verbena officinalis L. & glycosides, essential oil, mucus & herba \\
\hline \multirow[t]{6}{*}{ Lamiaceae } & Ajuga reptans $\mathrm{L}$. & tannins, phenolic acids & herba \\
\hline & Teucrium chamaedrys L. * & essential oil, tannins, flavonoids & herba \\
\hline & Teucrium montanum L. * & $\begin{array}{l}\text { bitter substance, tannins, mineral } \\
\text { substances, essential oil }\end{array}$ & herba \\
\hline & Teucrium polium L. & essential oil, tannins & herba \\
\hline & Marrubium vulgare L. * & lactone marubin, essential oil, tannins & herba \\
\hline & Nepeta cataria $\mathrm{L}$. & $\begin{array}{l}\text { essential oil, tannins, saponins, } \\
\text { glycosides }\end{array}$ & herba \\
\hline
\end{tabular}




\begin{tabular}{|c|c|c|c|}
\hline Family & Taxon & Compounds & Herbal drug \\
\hline & Nepeta nuda L. & $\begin{array}{l}\text { essential oil, tannins, saponins, } \\
\text { glycosides }\end{array}$ & herba \\
\hline & Glechoma hederacea L. & tannins, bitter substance, essential oil & herba \\
\hline & Glechoma hirsuta Waldst. \& Kit. & bitter substance, tannins, essential oil & herba \\
\hline & Prunella vulgaris L. & tannins, flavonoids, saponins & herba \\
\hline & Melittis melissophyllum L. & essential oil, coumarins, flavonoids & herba \\
\hline & Galeopsis pubescens Besser & bitter substance, tannins, glycosides & herba \\
\hline & Galeopsis speciosa Miller & tannins, saponins & herba \\
\hline & Lamium purpurerum $\mathrm{L}$. & $\begin{array}{l}\text { saponins, mucus, flavonoids, essential } \\
\text { oil }\end{array}$ & herba \\
\hline & Leonurus cardiaca L. * & glycosides, alkaloids, tannins, saponins & herba \\
\hline & Ballota nigra $\mathrm{L}$. & bitter substance, tannins, essential oil & herba \\
\hline & Stachys officinalis (L.) Trevisan & tannins, glycosides, saponins & herba \\
\hline & Stachys sylvatica $\mathrm{L}$ & tannins, saponins & herba \\
\hline & Stachys recta $\mathrm{L}$. & tannins, saponins & herba \\
\hline & Salvia glutinosa $\mathrm{L}$. & essential oil, bitter substance & folium \\
\hline & Salvia sclarea L. & essential oil, bitter substance & folium \\
\hline & Salvia pratensis L. & essential oil, bitter substance & folium \\
\hline & Salvia nemorosa $\mathrm{L}$. & essential oil, bitter substance & folium \\
\hline & Melissa officinalis $\mathrm{L}$. & essential oil, flavonoids & folium \\
\hline & Satureja kitaibelii Wierzb. * & essential oil & herba \\
\hline & Calamintha officinalis Moench & tannins, bitter substance, essential oil & herba \\
\hline & Calamintha nepeta (L.) Savi & essential oil & herba \\
\hline & Hyssopus officinalis L. & essential oil, flavonoids, tannins & herba \\
\hline & Origanum vulgare L. * & essential oil, tannins & herba \\
\hline & Thymus sp. div. & $\begin{array}{c}\text { essential oil, tannins, bitter substance, } \\
\text { flavonoids }\end{array}$ & herba \\
\hline & Lycopus europaeus L. & bitter substance, tannins & herba \\
\hline & Mentha piperita $\mathrm{L}$. & essential oil, tannins, flavonoids & folium \\
\hline & Mentha pulegium $\mathrm{L}$. & essential oil, tannins, bitter substance & herba \\
\hline Campanulaceae & Campanula glomerata $\mathrm{L}$. & not studied & herba \\
\hline & Campanula rapunculus $\mathrm{L}$. & inulin & herba, radix \\
\hline & Campanula trachelium $\mathrm{L}$. & not studied & herba \\
\hline Asteraceae & Eupatorium cannabinum L. & essential oil, resin, tannins & radix, herba \\
\hline & Solidago virgaurea L. *! & $\begin{array}{c}\text { tannins, flavonoids, saponins, essential } \\
\text { oil }\end{array}$ & herba \\
\hline & Bellis perennis $\mathrm{L}$. & bitter substance, tannins, saponins & $\begin{array}{l}\text { herba, flos, } \\
\text { folium }\end{array}$ \\
\hline & Telekia speciosa (Schreber) Baumg. & essential oil, bitter compounds, inulin & radix \\
\hline & Achillea clypeolata Sibth. \& Sm. * & tannins & folium, flos \\
\hline & Achillea crithmifolia Waldst. \& Kit. & bitter substance, essential oil & $\begin{array}{l}\text { herba, flos, } \\
\text { folium }\end{array}$ \\
\hline & Achillea millefolium L. * & bitter substance, essential oil & $\begin{array}{l}\text { herba, flos, } \\
\text { folium }\end{array}$ \\
\hline & Matricaria chamomilla $\mathrm{L}$. & $\begin{array}{c}\text { essential oil, flavonoids, coumarins, } \\
\text { mucus }\end{array}$ & flos \\
\hline & Tanacetum vulgare $\mathrm{L}$. & $\begin{array}{l}\text { essential oil, flavonoids, tannins, bitter } \\
\text { compounds }\end{array}$ & flos, herba \\
\hline & Artemisia vulgaris $\mathrm{L}$. & essential oil, bitter substance, tannins & herba \\
\hline & Artemisia pontica L. * & essential oil, bitter substance & herba \\
\hline & Artemisia alba Turra & $\begin{array}{l}\text { essential oil, flavonoids, bitter } \\
\text { substance }\end{array}$ & herba \\
\hline & Tussilago farfara $\mathrm{L}$. & mucus, inulin, tannins, flavonoids & flos, folium \\
\hline
\end{tabular}




\begin{tabular}{|c|c|c|c|}
\hline Family & Taxon & Compounds & Herbal drug \\
\hline & $\begin{array}{c}\text { Petasites hybridus (L.) P. Gaertn., B. } \\
\text { Mey. \& Scherb. * }\end{array}$ & $\begin{array}{l}\text { essential oil, flavonoids, mucus, } \\
\text { tannins }\end{array}$ & radix, folium \\
\hline & Carlina acaulis L. $*$ & inulin, essential oil, tannins, resin & radix \\
\hline & Arctium tomentosum Miller & $\begin{array}{l}\text { inulin, mucus, essential oil, fatty oil, } \\
\text { tannins }\end{array}$ & radix \\
\hline & Arctium lappa L. * & $\begin{array}{l}\text { inulin, mucus, essential oil, fatty oil, } \\
\text { tannins }\end{array}$ & radix \\
\hline & Centaurea cyanus L. & anthocyanins, glycosides, tannins & flos \\
\hline & Cichorium intybus $\mathrm{L}$. & inulin, glycosides, tannins, pectins & herba, radix \\
\hline & Taraxacum officinale Weber & glycosides, resin, mucus, inulin & radix, herba \\
\hline & Hieracium pilosella $\mathrm{L}$. & $\begin{array}{l}\text { tannins, flavonoids, coumarins, resin, } \\
\text { mucus }\end{array}$ & $\begin{array}{l}\text { herba cum } \\
\text { radicibus }\end{array}$ \\
\hline Liliaceae & Veratrum album L. & alkaloids & rhizoma \\
\hline & Veratrum nigrum L. * & alkaloids & rhizoma \\
\hline & Colchicum autumnale L. *! & alkaloids & semen, tuber \\
\hline & Lilium martagon L. * & mucus, essential oil & $\begin{array}{l}\text { folium, flos, } \\
\text { bulbus }\end{array}$ \\
\hline & Allium ursinum L. * & $\begin{array}{l}\text { sulfuric essential oil, vitamins, mineral } \\
\text { substances }\end{array}$ & $\begin{array}{l}\text { herba recens, } \\
\text { bulbus recens }\end{array}$ \\
\hline & Convallaria majalis L. ! & $\begin{array}{l}\text { cardiotonic glycosides, saponins, } \\
\text { flavonoids }\end{array}$ & $\begin{array}{l}\text { herba, folium, } \\
\text { flos }\end{array}$ \\
\hline Amarylidaceae & Galanthus nivalis L. *! & choline, phytosterol & $\begin{array}{l}\text { bulbus, folium, } \\
\text { flos }\end{array}$ \\
\hline Asparagaceae & Asparagus officinalis L. & asparagine, saponins & rhizoma, radix \\
\hline Iridaceae & Crocus tommasinianus Herbert! & alkaloids, carotenoids & stigma \\
\hline & Crocus adamii Gay! & alkaloids, carotenoids & stigma \\
\hline Dioscoreaceae & Tamus communis L.! & $\begin{array}{c}\text { alkaloids, starch, mucus, calcium } \\
\text { oxalate, saponins }\end{array}$ & rhizoma recens \\
\hline Orchidaceae & Orchis morio L. * & mucus & tuber \\
\hline & Orchis simia Lam. * & mucus, starch & tuber \\
\hline & Orchis militaris L. ** & mucus, starch & tuber \\
\hline & Orchis laxiflora Lam. ** & mucus & tuber \\
\hline & $\begin{array}{l}\text { Anacamptis pyramidalis (L.) L. C. M. } \\
\text { Richard * }\end{array}$ & mucus, starch, proteins & tuber \\
\hline & Gymnadenia conopsea (L.) R.Br. * & mucus, starch & tuber \\
\hline & $\begin{array}{c}\text { Platanthera bifolia (L.) L. C. M. Richard } \\
*\end{array}$ & mucus, starch & tuber \\
\hline & $\begin{array}{c}\text { Platanthera chlorantha (Custer) } \\
\text { Reichenb. } * *\end{array}$ & mucus, starch & tuber \\
\hline Poaceae & Elymus repens (L.) Gould & $\begin{array}{l}\text { carbohydrates, mucus, sugar alcohols, } \\
\text { saponins }\end{array}$ & rhizoma \\
\hline Araceae & Arum maculatum L.! & starch & rhizoma \\
\hline
\end{tabular}

\section{Legend:}

* - protected species (Prilog II) according to National legislative "Službeni glasnik Republike Srbije br. 5/2010, 47/2011, 32/2016, 98/2016 ; ** - strictly protected species (Prilog I) according to National legislative Službeni glasnik Republike Srbije br. 5/2010, 47/2011, 32/2016, 98/2016);

! - plant species with content of toxic substances.

\section{NUMERICAL RESULTS}

We are noted 264 plants on Vidlič Mt. that are considered medicinal according to Sarić ed. (1989) in our country (tab. 1.). The group of medicinal plants in Serbia includes 420 species (Sarić, 1989). Complete taxonomic analysis of the medicinal flora at Svrljiški Timok gorge in Eastern Serbia was done by Zlatković \& Bogosavljević (2014). They found that the flora of medicinal plant at Svrljiški Timok gorge includes 190 taxa.

Families that contain the largest number of plants on Vidlič Mt. are: Lamiaceae (33), Rosaceae (23), Asteraceae (21), Ranunculaceae (13), Apiaceae (11), Scrophulariaceae (10). Similar results about number of medicinal taxa per 
families were found Zlatković \& Bogosavljević (2014) in the flora of Svrljiški Timok, where the families with the greatest number of medicinal representatives were Lamiaceae (32 taxa), Asteraceae (20 taxa) and Rosaceae (14 taxa). In the flora of Serbia families that contain the largest number of taxa are also Lamiaceae (16,2\%), Asteraceae (12\%) and Rosaceae (9\%) (Sarić, 1989). The results about utilization of medicinal plants from different families were found by Zlatković et al. (2014) at Mt. Rtanj in Eastern Serbia. According to their results the plants from families Lamiaceae (22\%), Rosaceae (20\%) and Asteraceae $(30 \%)$ are most comonly used in traditional medicine.

Aerial herbose part (herba) is most commonly used for medicinal purposes (112 plants). Leave (folium) is used from 70 plants, root (radix) from 33 plants, flower or inflorescence (flos) from 29 plants, fruit (fructus) from 28 plants. Review of plant parts that are medicinal with the number of taxa is given in the table 2 .

The largest number of medicinal plants contains tannins as active medicinal substances (118 plants), followed by plants with content of flavonoids (80 plants), essential oils (67 plants), saponins (49 plants), glycosides (38 plants), mucus (37 plants), alkaloids (32 plants), bitter substances (32 plants) vitamins (19 plants) (Graph. 1).

Table 2. Review of herbal drugs with the number of taxa recorded on Vidlič Mt.

\begin{tabular}{|l|c|}
\hline Medicinal parts of plants & Number of plants \\
\hline herba - aerial herbose part & 112 \\
\hline folium - leave & 70 \\
\hline radix - root & 33 \\
\hline flos - flower or inflorescence & 29 \\
\hline fructus - fruit & 28 \\
\hline cortex - bark & 24 \\
\hline rhizoma - rhizome & 20 \\
\hline semen - seed & 2 \\
\hline tuber - tuber & 2 \\
\hline succus - sap & 4 \\
\hline stipes - stem & 2 \\
\hline gemma, turio - bud & \\
\hline bulbus - bulb & \\
\hline stigma - stigma & \\
\hline petiolus - stalks & \\
\hline
\end{tabular}

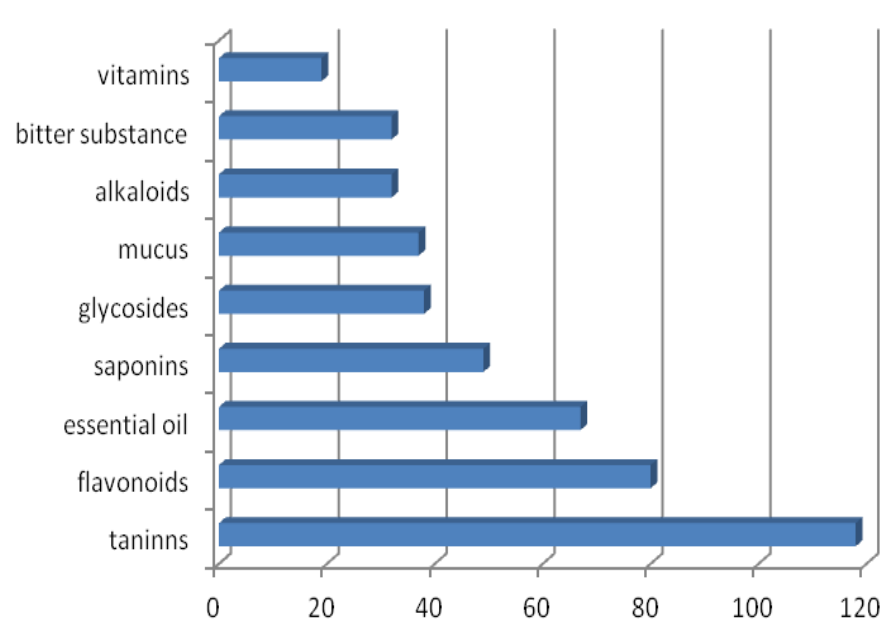

Figure 1. Review of dominant active substances with the number of medicinal plants of the Vidlič Mountain.

Particular attention was given to the plants with content of toxic substances, as well as rare and protected species.

42 plant species $(15,91 \%$ of the total number of species $)$ contain very toxic substances, so caution is advised in their use.

55 plant species $(20,83 \%)$ are protected, and 9 species $(3,41 \%)$ have become so rare with irrational exploatation, and they are strictly protected, with regulation named „Pravilnik o proglašenju i zaštiti strogo zaštićenih i zaštićenih divljih vrsta biljaka, životinja i gljiva“" (Službeni glasnik Republike Srbije br. 5/2010, 47/2011, 32/2016, 98/2016) Strictly protected species recorded on the Vidlič Mt. (Pulsatilla montana subsp. bulgarica, Adonis vernalis, Paeonia tenuifolia, Paeonia peregrina, Vinca herbacea, Menyanthes trifoliata, Orchis militaris, Orchis laxiflora and Platanthera chlorantha) are considered as threatended taxons of flora in Serbia, and almost exclusively, cannot be collected and used any purpose (Stevanović ed, 1999).

\section{CONCLUSION}

In comparison with several well done, published studies on medicinal flora in surrounding limestone areas of southeastern Serbia, medicinal flora on Vidlič Mt. is very rich and diverse, that includes 264 medicinal plant species. Family Lamiaceae is the richest with medicinal plants. Aerial herbose parts of plants in bloom are produced mostly. The largest number of medicinal plant species of the Vidlič Mt. as a core group of active ingredients containing tannins.

Toxic substances in higher concentrations are registred in $15,91 \%$ of analyzed species. Some representatives of the medicinal plants of Vidlič Mt. are strictly protected $(3,41 \%)$, and $20,83 \%$ species are protected. 


\section{ACKNOWLEGMENTS}

This work is a result of the project „Ethno pharmacological study of the region of Southeastern Serbia“. This project is supported by the Serbian Academy of Sciences and Arts. The authors owe special gratitude to academician dr Dragoslav Marinković, head of the project.

\section{REFERENCES}

Anđelković, M., \& Nikolić, P. 1958. Geološki sastav i tektonika jugozapadnih padina Stare planine. Beograd: SKANj. knj. CCCXVII.

Antonović, G., \& Mrvić, V. 2008. Zemljišta sliva Nišave. Beograd, Srbija: Institut za zemljište.

Ćirić, J. 1971. Geografski položaj i granice Pirota i Pirotskog Regiona. Pirotski zbornik, 3, pp. 21-31.

Ćirić, J. 1989. Geografija gornjeg Ponišavlja i Lužnice. In P. Nikolić Ed., Pirotski zbornik.Pirot: Novinsko izdavačka ustanova \"Slobodal". 16, pp. 9-23.

Josifović, M., Ed. 1970. Flora SR Srbije. Beograd: Srpska Akademija Nauka i Umetnosti. 1970-1986; I-X.

Marinkov, J. 1999. Zabrdje, prirodno-privredni potencijali. Dimitrograd: Narodna biblioteka.

Marković, M. 2006. Prirodni potencijali spontane aromatične lekovite flore planine Vidlič. Univerzitet u Kragujevcu Prirodno matematički fakultet - Institut za biologiju i ekologiju. magistarska teza.

Marković, M., Pavlović-Muratspahić, D., Matović, M., Markovic, A., \& Stankov-Jovanović, V. 2009. Aromatic Flora of the Vidlič Mountain. Biotechnology \& Biotechnological Equipment, 23(2), pp. 1225-1229. doi:10.1080/13102818.2009.10817643

Marković, M., Matović, M., Pavlović, D., Zlatković, B., Marković, A., Jotić, B., \& Stankov-Jovanović, V. 2010a. Resources of medicinal plants and herbs collector's calendar of Pirot County (Serbia). Biologica nyssana, 1(12), pp. 9-21.

Marković, M., Matović, M., Pavlović, D., Zlatković, B., Marković, A., Jotić, B., \& Stankov-Jovanović, V. 2010b. Biljarski kalendar subregiona Pirot. In 10th Symposium on the Flora of Southeastern Serbia and Neighbouring regions, Vlasina, 17 to 20 june. Book of abstracts, 103.

Marković, M. 2013. Sukcesije biljnih zajednica na požarištima planine Vidlič, Phd thesis, Institut za biologiju i ekologiju, Prirodno matematički fakultet, Univerzitet u Kragujevcu, 20 june 2010, Book of abstracts, 103.

Marković, S. M., Nikolić, M. B., Zlatković, K. B., Nikolić, S. D., Rakonjac, B. Lj., Stankov-Jovanović, P. V., Djokić, M.
M., Ratknić, B. M., Lučić, Ž. A. 2018. Short-term patterns in the post-fire diversity of limestone grasslands and rocky ground vegetation, Applied Ecology and Environmental Research 16(3), pp. 3271-3288.

DOI: http://dx.doi.org/10.15666/aeer/1603_3271328

Martinović, Ž. 1979. Vrela i izvori u zabrdskom delu Vidliča prilog poznavanju podzemnih voda istočne Srbije. Zbornik radova PMF Priština, 1979-1980, VI, pp. 283-300.

Mišić, V., Jovanović-Dunjić, R., Popović, M., Borisavljević, Lj., Antić, M., Danon, J., \& Blaženčiž, Ž. 1978. Biljne zajednice i staništa Stare planine. Beograd: Srpska Akademija Nauka i Umetnosti.

Ranđelović, N., Sotirov, S., Stamenković, V., Rexhepi, F., Ranđelović, V., \& Zlatković, B. 1991. Lekovito biqe subregiona Pirot. Zbornik radova Tehnološkog fakulteta $\mathrm{u}$ Leskovcu, 7/8, pp. 29-33.

Sarić, M. 1989. Lekovite biljke SR Srbije.Beograd: Srpska Akademija Nauka i Umetnosti - Odeljenje prirodnomatematičkih nauka. Posebna izdanja DXCVIII.

Službeni glasnik Republike Srbije 2010. Pravilnik o proglašenju i zaštiti strogo zaštićenih i zaštićenih divljih vrsta biljaka, životinja i gljiva. Službeni glasnik Republike Srbije, . br. 5/10, 47/11 i 32/16.

Stevanović, V., Ed., 1999. Crvena knjiga flore Srbije 1 iščezli i krajnje ugroženi taksoni.Beograd: Ministarstvo za životnu sredinu Republike Srbije / Univerzitet u Beogradu - Biološki fakultet / Zavod za zaštitu prirode Republike Srbije.

Tutin, T.G., Heywood, V.H. Burges, N.A., Moore, D.M., Valentine, D.H., Walters, S.M. Webb, D.A., Eds. 1964. Flora Europaea.London: Cambridge University Press. 1964-1980, I-V.

Tutin, T.G., Burges, N.A., Chater, O.A., Edmondson, J.R., Heywood, V.H., Moore, D.M., Valentine, D.H., Walters, S.M., Webb D.A. Eds. 1993. Flora Europaea. London: Cambrige University Press. 1 (2nd Edition).

Vidanović, G. 1955. Visok, privredno geografska ispitivanja.Beograd: Srpska akademija nauka / Geografski institut. Posebna izdanjač knj. 6.

Zlatković, B., Bogosavljević, S., Radivojević, A., Pavlović, M. 2014. Traditional use of the native medicinal plant resource of Mt. Rtanj (Eastern Serbia): ethnobotanical evaluation and comparation., Journal of Etnopharmacology 151(1), pp. 704-713. doi: 10.1016/j.jep.2013.11.037

Zlatković, B., Bogosavljević, S. 2014. Taxonomical and pharmacological valorization of medicinal flora in Timok gorge (Eastern Serbia), Facta Universitatis, Series: Medicine and Biology, 16(2), pp. 76-86. 